\title{
OBSERVATIONS OF BERYLLIUM IN STARS
}

\author{
AnN Merchant BoesGaARd
}

Since a considerably higher temperature is necessary to consume Be atoms by $(p, \alpha)$ reactions than Li atoms, it has been thought that many clues to the understanding of the synthesis and destruction of the light elements could be found by studying $\mathrm{Be}$ abundances and the $\mathrm{Li} / \mathrm{Be}$-abundance ratios. Calculations have been made by Bodenheimer (1966) to determine the amount of convective depletion of Be during pre-mainsequence evolution. He found that a star reaching the main sequence at $\mathrm{K} 5$ would have destroyed $15 \%$ of its original Be; a K2 dwarf would have depleted its Be by $2 \%$. For early $\mathrm{K}$ stars main-sequence depletion subsequent to this would be very slight. If there are no complicating effects such as magnetic fields and unusual patterns of circulation of stellar material, the observed Be abundances in stars of type $G$ and earlier should be the initial pre-stellar or proto-star abundances. The BeII resonance lines lie far in the ultraviolet at $\lambda 3131$. But despite the very difficult nature of the

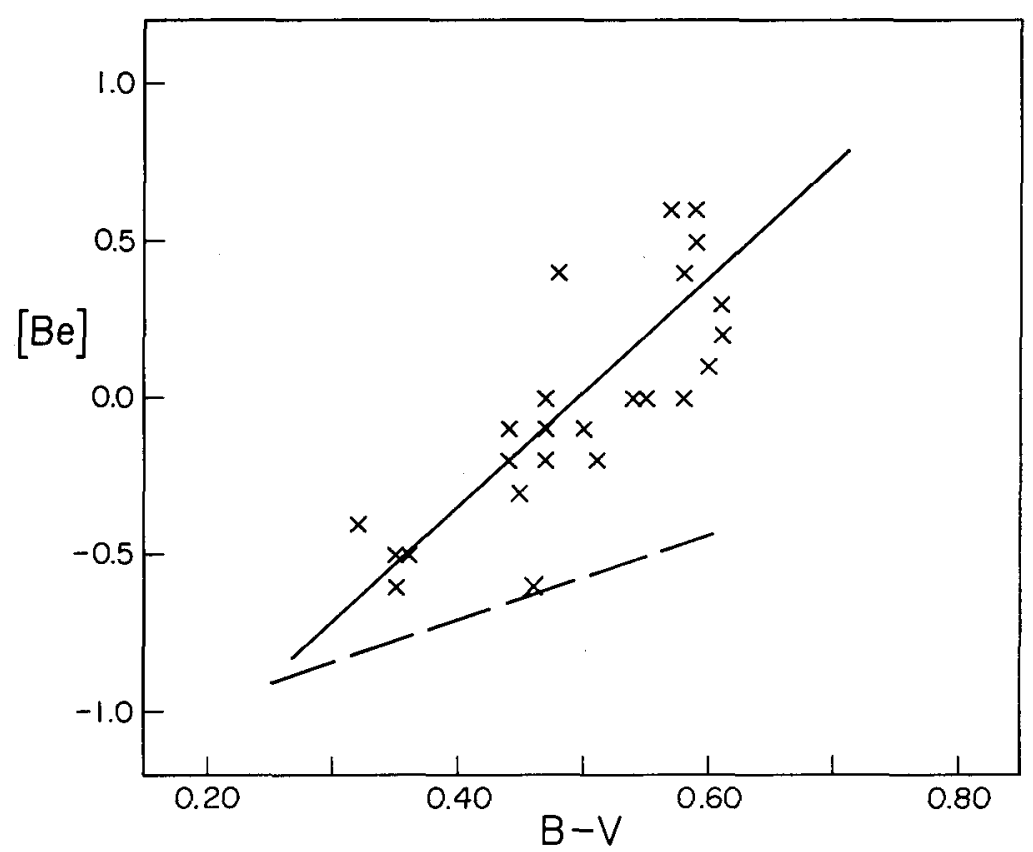

FIG. 1. The logarithmic abundance ratio of stellar beryllium to solar beryllium as a function of $B-V$ for dwarf stars only.

Perek (ed.), Highlights of Astronomy, 237-242. (c) I.A.U. 
observations, there are now Be abundances determined in about $60 \mathrm{dwarfs}$ and subgiants of types $\mathrm{A}, \mathrm{F}$, and $\mathrm{G}$ and in $2 \mathrm{~K}$ giants.

I shall now review these Be observations. Bonsack (1961) has observed 2 normal A stars to have solar Be, while Sirius had 40 times less Be than the Sun - perhaps due to its white-dwarf companion in some way -, and $\alpha^{2} \mathrm{CVn}$, a magnetic variable had 25 times the solar Be. In those peculiar A stars in which blending is not a serious problem, Sargent et al. (1962) have found 4 out of 15 stars to have strong Be lines and estimate the Be abundance in these four stars to be 100 times the solar value. The Be-rich stars are all Mn peculiar A stars, and all have abnormally strong PII lines.

In the stars of spectral type F-G both $\mathrm{Li}$ and Be can be observed. Now that there are Be observations in close to 30 of these dwarfs, a very interesting trend shows up, which can be seen in Figure 1. Here the $\log$ of the beryllium abundances relative to the Sun are plotted as a function of B-V for dwarf stars only. The dashed line represents the observational limit. Values on or below this line can only be upper limits. The three stars which gave only upper limits are not shown here. The points are a combination of observations by Conti and Danziger (1966), by Conti (1968), and by myself (Merchant, 1966). This reverse correlation is not what would have been anticipated and it seems that it can not be explained away as observational error or inappropriate ionization correction. The hotter stars should show stronger Bell lines for a given abundance than the cool ones - but in many cases the reverse is observed. The posi-

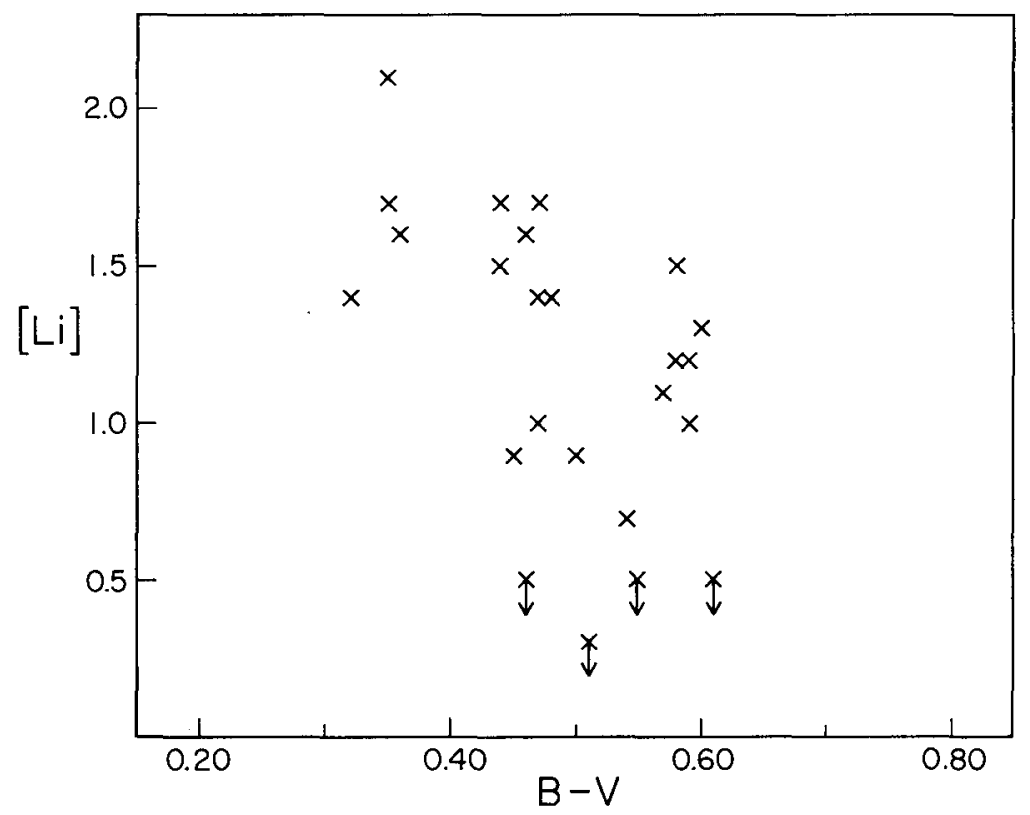

FIG. 2. The logarithmic abundance ratio of stellar lithium to solar lithium as a function of $B-V$ for the same stars as in Figure 1. 
tioning of the continuum was done with consistency, and the UV blending and blanketing does not change much over this B-V range in this wavelength region.

Since it is thought that ordinary convective depletion will affect cooler stars more than hotter stars, we must either look for a new depletion mechanism or postulate variations in initial composition which might be a function of mass. In the latter category, perhaps the cooler stars had more violent activity leading to more spallation reactions or a longer time during which the $\mathrm{Li}, \mathrm{Be}, \mathrm{B}$ producing activity took place. The energy spectrum of the bombarding protons could be different.

Figure 2 shows the $\mathrm{Li}$ observations in these same stars. Here there is more scatter but the trend is clearly in the opposite direction. If all these stars started out with the same $\mathrm{Li} / \mathrm{Be}$ ratio and nothing has happened to the $\mathrm{Be}$, then the $\mathrm{Li}$ depletion has been more severe than previously thought. If the energy spectrum of the bombarding protons is variable as a function of stellar mass, then the $\mathrm{Li} / \mathrm{Be}$ ratio would not necessarily be constant. Perhaps the theoreticians or experimentalists can shed some light on these alternatives.

Figure 3 shows an H-R diagram adapted from recent work by Conti (1968). The different symbols represent various ranges of values of the $\mathrm{Li} / \mathrm{Be}$ ratio. These are nonlogs. The Sun is at $\mathrm{B}-\mathrm{V}=0.62, M_{v}=4 \cdot 8$ with $\mathrm{Li} / \mathrm{Be}=0.08$. To the left of the vertical line there is no Li dilution according to Iben's calculation. By the time the star reaches the vertical dashed line, eighty-five per cent of the Li is diluted. So with the exception of $\delta$ Eri (at $\mathrm{B}-\mathrm{V}=0.93, M_{v}=3.7$ ) there is no dilution effect in these stars. Conti interprets the distribution of $\mathrm{Li} / \mathrm{Be}$ points on this $\mathrm{H}-\mathrm{R}$ diagram as due to an age effect. The timescale for main-sequence $\mathrm{Li}$ burning for the $\mathrm{G}$ stars is $5 \times 10^{7}-5 \times 10^{8}$ years determined from observations in the Pleiades and the Hyades. Since so few stars lying to the right of the Hyades track show appreciable Li destruction, Conti concludes that the timescale for $\mathrm{Li}$ burning in the main-sequence $\mathrm{F}$ stars is $5 \times 10^{8}$ to $5 \times 10^{9}$ years. That is, the destruction rate is inversely dependent on temperature or mass.

In summarizing the main features of the Be observations we can say:

(1) The peculiar A stars are peculiar in their Be content too, but the Mn stars have a greater tendency for high Be abundance than other A stars.

(2) The Be abundances in F and $\mathrm{G}$ dwarfs are inversely correlated with temperature. This may be due to:

(a) a new depletion mechanism,

(b) difference as a function of stellar mass in the length of time or intensity of the spallation activity, and/or

(c) difference as a function of stellar mass in the energy spectrum of the bombarding protons.

I have been asked to make some statement on the observability of boron. Boron so far has had an uncertain history. The infrared B I feature at $\lambda 8668$ observed in the Sun is thought to be $\mathrm{CN}$; the BIII suggested identification on $\mathrm{O}$ stars is now thought unlikely; the BH identification in the solar disk is probably in error although $\mathrm{BH}$ may 


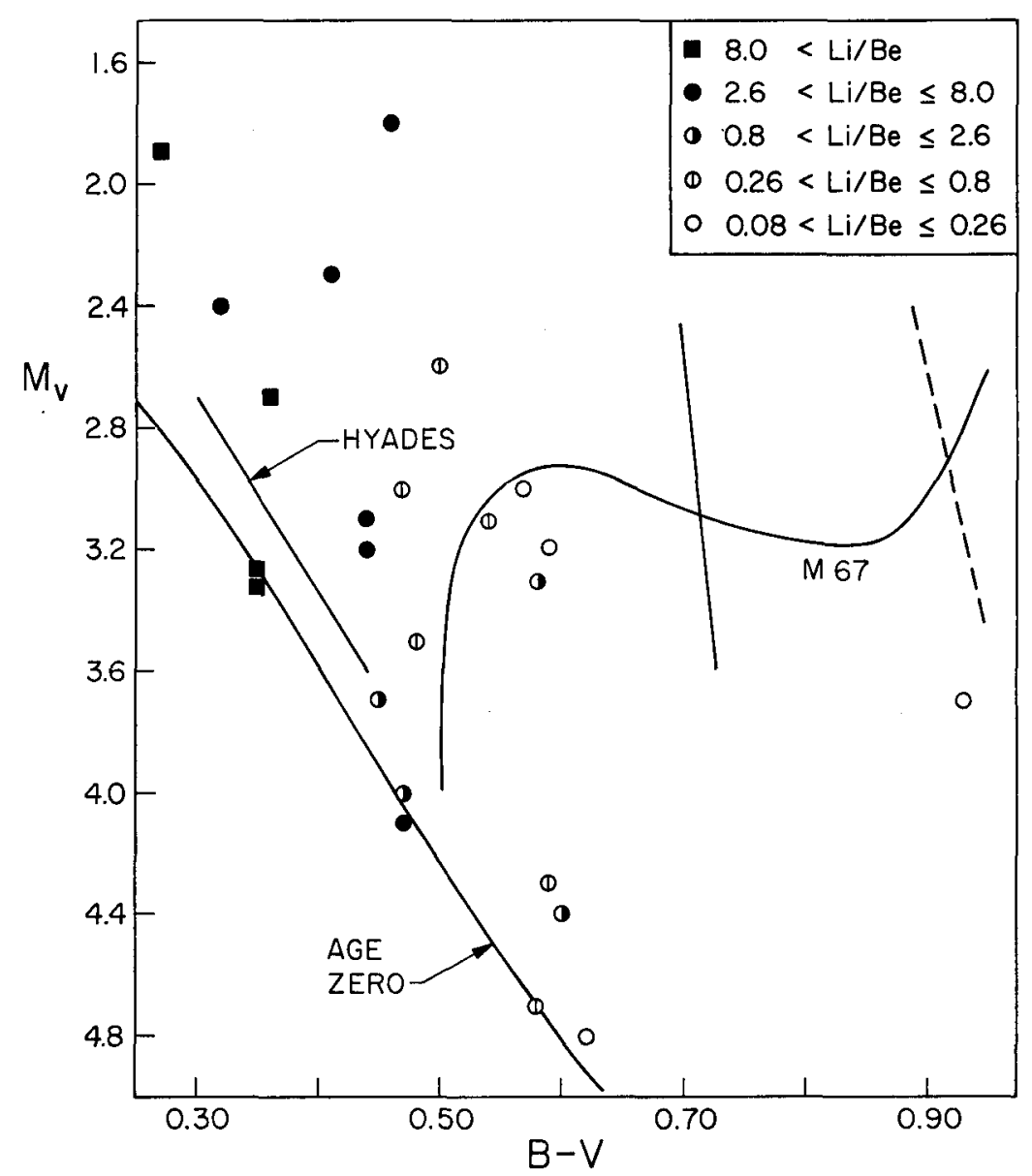

FIG. 3. An $H-R$ diagram in which the stars with positive observations of lithium and beryllium are shown with symbols corresponding to their Li/Be abundance ratio. The zero-age main sequence, the Hyades, and $M 67$ tracks are shown.

be present in sunspots. The 0,0 band of $\mathrm{BO}$ at $\lambda 4227$ has been suggested in $\beta$ Peg by Davis (1947). The small Frank-Condon factor for this band, 0.04, and the expected low abundance of $\mathrm{B}$ perhaps make this identification questionable. $\mathrm{But} \mathrm{BO}$ and $\mathrm{BO}_{2}$ should certainly be looked for in stars.

There are two possible observations of atomic B: a high-excitation line of BII occurs at $\lambda 3451.4$ and might appear in late B and A stars. However, it is quite badly blended with 2 lines of FeII. Perhaps the best chance for B observations is in the rocket ultraviolet where lines of $\mathrm{Br}$ occur at $\lambda 2497.7$ and $\lambda 2496.8 \AA$. Observations made so far give upper limits for the sun. This would be a profitable region to observe since nearby at $\lambda 2494.6$ are 3 lines of BeI. The Be lines are present, but weak and unresolved by observations so far of the sun. 


\section{References}

Bodenheimer, Peter (1966) Astrophys. J., 144, 103.

Bonsack, Walter K. (1961) Astrophys. J., 133, 551.

Conti, Peter (1968) Astrophys. J., 151, 567.

Conti, Peter, Danziger, I. J. (1966) Astrophys. J., 146, 383.

Davis, D. N. (1947) Astrophys. J., 106, 28.

Merchant, Ann (1966) Astrophys. J., 143, 336.

Sargent, W.L.W., Searle, L., Jugaku, J. (1962) Publ. Astron. Soc. Pacific, 74, 408.

\section{DISCUSSION}

Underhill: The fact that standard spectrum analyses of $\mathrm{Li}$ and Be lines give a variety of abundances, whereas those for $\mathrm{Na}$ or Fe give normal abundances in many stars, may possibly be affected by the particular intensity distribution in the far UV and X-ray regions of these stars. $\mathrm{Li}$ and $\mathrm{Be}$ are elements 3 and $4, \mathrm{Na}$ is element 11 ; in the latter case many electrons must be removed and perhaps the distribution of jonic species follows the predictions from Saha's Law better than in the case of $\mathrm{Li}$ or Be with only few electrons to remove and thus possibly more sensitive to a particular line emission spectrum in the far UV.

Conti (in answer to Dr. Underhill): It seems to me more than a coincidence that it is just those light elements, $\mathrm{Li}$ and $\mathrm{Be}$, which are easily destroyed in $\mathrm{F}$ and $\mathrm{G}$ stars, that are anomalous. In fact, they are the only greatly anomalous elements in these stars. Rather than interpret these anomalies in terms of non-LTE, it seems more realistic to consider them as nuclear processes.

Müller: Concerning the B abundance in the solar atmosphere I should like to mention that D. L. Lambert, E.A. Mallia, and B. Warner recently gave an upper limit of $4 \times 10^{-9}$ derived from the infrared BI line at $\lambda 11662.47$ that might be present. They stress, however, that the solar $B$ abundance may be an order of magnitude smaller. The best way to determine the solar boron abundance would be to study the BH bands in sunspots.

Delbouille: As a comment to Dr. E. Müller's remark, we can say that, from observations made by Dr. Roland and myself at the Jungfraujoch of the possible B line at $\lambda 11662 \AA$, Grevesse and Boury conclude that $\mathrm{B}<2.5$ on $\log H=12.00$ scale.

Müller: Concerning the solar Be abundance, I should like to point out that the BeI and BeII lines used in the abundance determination fall in the wavelength region which (1) is very crowded and (2) is way down in the UV (around $\lambda 3100-3300 \AA$ ) where the continuum is highly uncertain. Consequently, the solar beryllium is, for the moment, still quite doubtful.

Boury: We have redetermined solar Be abundances through Be $\mathrm{I}$ and BeII lines. We find, on the $\log N_{\mathrm{H}}=12.00$ scale, 2.33 from BeI lines and only 1.24 from BeI lines. BeI lines are very weak and much perturbed.

Reeves: What is the definition here of Be solar units?

Boesgaard: We used Mutschlecner's determination of 6.9 Be atoms per $10^{6} \mathrm{Si}$ atoms. This number can be readily converted back to the $\log H=12.00$ scale through the GMA value for Si.

Aller: It is not probable that a reliable solar $\mathrm{Li}$ abundance can be obtained unless one takes into account the line profiles - and more particularly the detailed energy distribution in the spectrum. One must 'synthesize' the spectrum by computing the superposed absorption by the continuum and many lines of different elemental origins and excitation potentials. Successful application of this procedure requires very accurate line-profile data and also centre-limb variations. It is necessary also to know the absolute flux in the solar spectrum at some point in your wavelength interval. The method has been applied by John Ross with encouraging results to lines of $\mathrm{Ag}$ and $\mathrm{Pb}$ in the solar spectrum.

Bidelman: I believe that it is likely that $\mathrm{B}_{\mathrm{II}}$ is present in the strange A-type star HR $6870=\mathrm{HD}$ 168733 , but Dr. Aller has been studying this star and may know more about this possibility.

Aller: The spectrum of this star is terribly crowded with unidentifiable lines. When Steve Little completes his measurements of the spectrum, we may be able to say something of more confidence about the $B$ spectrum. 
Boesgaard: Do you know anything about the BeII lines in that star?

Aller: The declination of $\mathrm{HR} 6870$ is $-36^{\circ}$, so the far UV is very difficult to observe at the latitude of Lick Observatory.

Reeves: Please look at upper limits of $\mathrm{B} / \mathrm{Be}$ ratios in stars. 